\title{
Faithful orthogonal representations of graphs from partition logics
}

\author{
Karl Svozil ${ }^{1}$ (1) \\ Published online: 4 November 2019 \\ (c) The Author(s) 2019
}

\begin{abstract}
Partition logics often allow a dual probabilistic interpretation: a classical one for which probabilities lie on the convex hull of the dispersion-free weights and another one, suggested independently from the quantum Born rule, in which probabilities are formed by the (absolute) square of the inner product of state vectors with the faithful orthogonal representations of the respective graph. Two immediate consequences are the demonstration that the logico-empirical structure of observables does not determine the type of probabilities alone and that complementarity does not imply contextuality.
\end{abstract}

Keywords Quantum mechanics · Gleason theorem · Kochen-Specker theorem · Born rule · Partition logic · GrötschelLovász-Schrijver set

\section{Partition logics as nonboolean structures pasted from Boolean subalgebras}

Partitions provide ways to distinguish between elements of a given finite set $\mathscr{S}_{n}=\{1,2, \ldots, n\}$. The Bell number $B_{n}$ (after Eric Temple Bell) is the number of such partitions (Sloane 2018). (Obvious generalizations are infinite denumerable sets or continua.) We shall restrict our attention to partitions with an equal number $1 \leq m \leq n$ of elements. Every partition can be identified with some Boolean subalgebra $2^{m}$ —in graph theoretical terms a clique — of $2^{n}$ whose atoms are the elements of that partition.

A partition logic (Svozil 1993; Schaller and Svozil 1994, 1996; Dvurečenskij et al. 1995; Svozil 2005) is the logic obtained (i) from collections of such partitions, each partition being identified with an $m$-atomic Boolean subalgebra of $2^{n}$, and (ii) by "stitching" or pasting these subalgebras through identifying identical intertwining elements. In quantum logic, this is referred to as pasting construction, and the partitions are identified with, or are synonymously denoted

Communicated by F. Holik.

Karl Svozil

svozil@tuwien.ac.at

http://tph.tuwien.ac.at/ svozil

1 Institute for Theoretical Physics, Vienna University of Technology, Wiedner Hauptstrasse 8-10/136, 1040 Vienna, Austria by, blocks, subalgebras or cliques, which are representable by orthonormal bases or maximal operators.

Partitions represent classical mini-universes which satisfy compatible orthogonality, or Specker's exclusivity principle (Specker 1960, 2009; Cabello 2013; Fritz et al. 2013; Henson 2012; Cabello 2012; Cabello et al. 2013, 2014): if any two observables corresponding to two elements of a partition are co-measurable, the entire set of observables corresponding to all elements of that partition are simultaneously measurable. (For Hilbert spaces, this is a well-known theorem; see, for instance, (von Neumann 1931, Satz 8, p. 221) and (von Neumann 1955, p. 173), or (Halmos 1958, § 84, Theorem 1, p. 171).)

Unlike complete graphs $K_{m}$ representations of $m$-vertex cliques in which every pair of distinct vertices is connected by a unique edge in quantum logic, it is quite common to conveniently depict cliques (aka contexts) as smooth curves, referred to as Greechie orthogonality diagram (Greechie 1971). For example, the two ad hoc partitions

$$
\{\{1\},\{2\},\{3,4\}\} \text { and }\{\{1\},\{3\},\{2,4\}\}
$$

of $\mathscr{S}_{3}=\{1,2,3,4\}$ form two 3-atomic Boolean algebras $2^{3}$ with one identical intertwining atom $\{1\}$, as depicted in Fig. 1a. It is the logic $L_{12}$ (because it has 12 elements) is just two straight lines (representing the two contexts or cliques) interconnected at $\{1\}$. (Cf. Fig. 1 of Wright's "Bowtie Example 3.1" (Wright 1990, pp. 884,885).)

Many partition logics, such as the pentagon logic, have quantum doubles. One of the (necessary and sufficient) crite- 


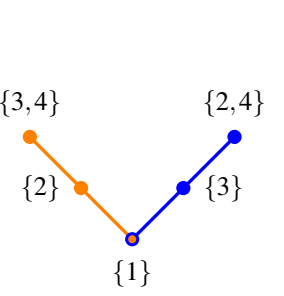

(a)

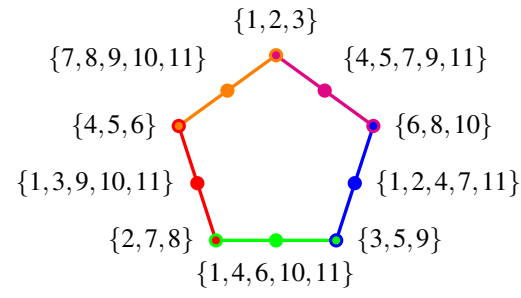

(b)
Fig. 1 Greechie orthogonality diagrams of a the $L_{12}$ logic and b the pentagon (pentagram) logic, with two of their associated (quasi)classical partition logic representations

ria for quantum logics to be representable by a partition logic is the separability of pairs of atoms of the logic by dispersionfree (aka two-valued, $\{0,1\}$-valued) weights/states (Kochen and Specker 1967, Theorem 0, p. 67), interpretable as classical truth assignments.

Conversely, "sufficiently" many (more precisely, a separating set of) dispersion-free states allow the explicit (re)construction of a partition logic (Svozil 2005, 2009). For instance, the five cyclically intertwined contexts/cliques forming a pentagon/pentagram logic (Ron 1978, p. 267, Fig. 2) support 11 dispersion-free states $v_{1}, \ldots, v_{11}$. Constructing 5 contexts/cliques from the occurrences of the dispersion-free value 1 on the respective 10 atoms results in the partition logic based on the set of indices of the dispersion-free states $\mathscr{S}_{11}=\{1, \ldots, 11\}$, as depicted in Fig. 1b

$\{\{\{1,2,3\},\{4,5,7,9,11\},\{6,8,10\}\}$,

$\{\{6,8,10\},\{1,2,4,7,11\},\{3,5,9\}\}$,

$\{\{3,5,9\},\{1,4,6,10,11\},\{2,7,8\}\}$,

$\{\{2,7,8\},\{1,3,9,10,11\},\{4,5,6\}\}$,

$\{\{4,5,6\},\{7,8,9,10,11\},\{1,2,3\}\}\}$.

According to this construction, the earlier logic $L_{12}$ would have a partition logic representation $\{\{\{2,3\},\{4,5\},\{1\}\},\{\{1\}$, $\{3,5\},\{2,4\}\}\}$ based on $\mathscr{S}_{5}=\{1, \ldots, 5\}$, corresponding to its 5 dispersion-free states.

\section{Probabilities on partition logics}

The following hypothesis or principle is taken for granted: probabilities and expectations on classical substructures of an empirical logic should be classical, that is, mutually exclusive co-measurable propositions (satisfying Specker's exclusivity principle) should obey Kolmogorov's axioms, in particular nonnegativity and additivity. Nonnegativity implies that all probabilities are nonnegative: $P\left(E_{1}\right), \ldots$, $P\left(E_{k}\right) \geq 0$. Additivity among (pairwise) mutually exclu- sive outcomes $E_{1}, \ldots, E_{k}$ means that the probabilities of joint outcomes are equal to the sum of probabilities of these outcomes, that is, within cliques/contexts, for $k \leq m$ : $P\left(E_{1} \vee \cdots \vee E_{k}\right)=P\left(E_{1}\right)+\cdots+P\left(E_{k}\right) \leq 1$. In particular, probabilities add to 1 on each of the cliques/contexts. Furthermore, Kolmogorov's axioms can be extended to configurations of more than one (classical) context by assuming that, relative to any atomic element of some context, the sum of the conditional probabilities of all atomic elements in any other context adds up to one (Svozil 2018).

At the moment, at least three such types of probabilities are known to satisfy Specker's exclusivity principle, corresponding to classical, quantum and Wright's "exotic" pure weights, such as the weight $\frac{1}{2}$ on the vertices of the pentagon (Ron $1978, \omega_{0}$, p. 68) and on the triangle vertices (Wright 1990, pp. 899-902) (the latter logic is representable as partition logic (Dvurečenskij et al. 1995, Example 8.2, pp. 420,421), but not in two- or three-dimensional Hilbert space). The former two "nonexotic" types, based on representations of mutually disjoint sets and on mutually orthogonal vectors, will be discussed later.

It is not too difficult to imagine boxes allowing input/output analysis "containing" classical or quantum algorithms, agents or mechanisms rendering the desired properties. For instance, a model realization of a classical box rendering classical probabilities is Wright's generalized urn model (Ron 1978; Wright 1990; Svozil 2006, 2014) or the initial state identification problem for finite deterministic automaton (Moore 1956; Svozil 1993; Schaller and Svozil 1995, 1996)_both are equivalent models of partition logics (Svozil 2005) featuring complementarity without value indefiniteness.

Specker's parable of the overprotective seer (Specker 1960; Liang et al. 2011, 2017; Svozil 2016) involving three boxes is an example for which the exclusivity principle does not hold (Tarrida 2014, Section 116, p. 40). It is an interesting problem to find other potential probability measures based on different approaches which are also linear in mutually exclusive events.

\subsection{Probabilities from the convex hull of dispersion-free states}

For nonboolean logics, it is not immediately evident which probability measures should be chosen. The answer is already implicit in Zierler and Schlessinger's 1965 paper on "Boolean embeddings of orthomodular sets and quantum logic". Theorem 0 of Kochen and Specker's 1967 paper (Kochen and Specker 1967) states that separability by dispersion-free states (of image $2^{1}=0,1$ ) for every pair of atoms of the lattice is a necessary and sufficient criterion for a homomorphic embedding into some "larger" Boolean algebra. In 1978, Wright explicitly stated (Ron 1978, p. 272) "that every urn weight is "classical," i.e., in the convex 
hull of the dispersion-free weights." In the graph theoretical context Grötschel, Lovász and Schrijver have discussed the vertex packing polytope $V P(G)$ of a graph $G$, defined as the convex hull of incidence vectors of independent sets of nodes (Grötschel et al. 1986). This author has employed dispersion-free weights for hull computations on the Specker bug (Svozil 2001) and other (partition) logics supporting a separating set of two-valued states.

Hull computations based on the pentagon (modulo pentagon/pentagram graph isomorphisms) can be found in Refs. Klyachko et al. (2008), Bub and Stairs (2009, 2010), Badziąg et al. (2011) (for a survey see Svozil 2018, Section 12.9.8.3). The Bub and Stairs inequality (Bub and Stairs 2009, Equation (10), p. 697) can be directly read off from the partition logic (2), as depicted in Fig. 1b, which in turn are the cumulated indices of the nonzero dispersion-free weights on the atoms: the sum of the convex hull of the dispersionfree weights on the 5 intertwining atoms (the "vertices" of the pentagon diagram) represented by the subsets $\{1,2,3\}$, $\{6,8,10\},\{3,5,9\},\{2,7,8\},\{4,5,6\}$ of $\mathscr{S}_{11}$ is

$$
\begin{aligned}
& \left(\lambda_{1}+\lambda_{2}+\lambda_{3}\right)+\left(\lambda_{6}+\lambda_{8}+\lambda_{10}\right)+\left(\lambda_{3}+\lambda_{5}+\lambda_{9}\right) \\
& +\left(\lambda_{2}+\lambda_{7}+\lambda_{8}\right)+\left(\lambda_{4}+\lambda_{5}+\lambda_{6}\right) \leq 2 \sum_{i=1}^{11} \lambda_{i}=2 .
\end{aligned}
$$

\subsection{Born-Gleason-Grötschel-Lovász-Schrijver type probabilities}

Motivated by cryptographic issues outside quantum theory, (Lovász 1979) has proposed an "indexing" of vertices of a graph by vectors reflecting their adjacency: the graphtheoretic definition of a faithful orthogonal representation of a graph is by identifying vertices with vectors (of some Hilbert space of dimension $d$ ) such that any pair of vectors are orthogonal if and only if their vertices are not orthogonal (Lovász et al. 1989; Parsons and Pisanski 1989). For physical applications (Cabello et al. 2010; Solís-Encina and Portillo 2015) and others have used an "inverse" notation, in which vectors are required to be mutually orthogonal whenever they are adjacent. Both notations are equivalent by exchanging graphs with their complements or inverses.

There is no systematic algorithm to compute the minimal dimension for a faithful orthogonal representation of a graph. Lovász (1979), Cabello et al. (2013) gave a (relative to entropy measures (Haemers 1979) "optimal" vector representation of the pentagon graph depicted in Fig. $1 \mathrm{~b}$ in three dimensions $\left[L_{12}\right.$ depicted in Fig. $1 \mathrm{a}$ is a sublogic thereof]: modulo pentagon/pentagram graph iso-

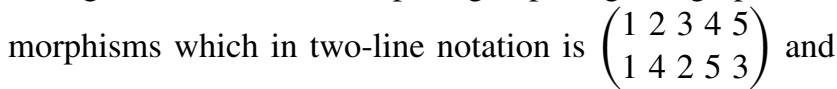
in cycle notation is (1)(2453); its set of five intertwining vertices $\left\{v_{1}, \ldots, v_{5}\right\}=\left\{u_{1}, u_{3}, u_{5}, u_{2}, u_{4}\right\}$ are represented by the three-dimensional unit vectors (the five vectors corresponding to the "inner" vertices/atoms can be found by a Gram-Schmidt process)

$\left|u_{l}\right\rangle=5^{-\frac{1}{4}}\left(1,\left[5^{\frac{1}{2}}-1\right]^{\frac{1}{2}} \cos \frac{2 \pi l}{5},\left[5^{\frac{1}{2}}-1\right]^{\frac{1}{2}} \sin \frac{2 \pi l}{5}\right)$,

which, by preparing the "(umbrella) handle" state vector $(1,0,0)$, turns out to render the maximal (Bub and Stairs 2009; Badzia̧g et al. 2011) quantum-bound $\sum_{j=1}^{5}\left|\left\langle c \mid u_{l}\right\rangle\right|^{2}=$ $\sqrt{5}$, which exceeds the "classical" bound (3) of 2 from the computation of the convex hull of the dispersion-free weights.

Based on Lovász's vector representation by graphs, Grötschel, Lovász and Schrijver have proposed (Grötschel et al. 1986, Section 3) a Gleason-Born type probability measure (Cabello 2019) which results in convex sets different from polyhedra defined via convex hulls of vectors discussed earlier in Sect. 2.1. Essentially their probability measure is based upon $m$-dimensional faithful orthogonal representations of a graph $G$ whose vertices $v_{i}$ are represented by unit vectors $\left|v_{i}\right\rangle$ which are orthogonal within, and nonorthogonal outside, of cliques/contexts. Every vertex $v_{i}$ of the graph $G$, represented by the unit vector $\left|v_{i}\right\rangle$, can then be associated with a "probability" with respect to some unit "preparation" (state) vector $|c\rangle$ by defining this "probability" to be the absolute square of the inner product of $\left|v_{i}\right\rangle$ and $|c\rangle$, that is, by $P\left(c, v_{i}\right)=\left|\left\langle c \mid v_{i}\right\rangle\right|^{2}$. Iff the vector representation (in the sense of Cabello-Portillo) of $G$ is faithful, the Pythagorean theorem assures that, within every clique/context of $G$, probabilities are positive and additive, and (as both $\left|v_{i}\right\rangle$ and $|c\rangle$ are normalized) the sum of probabilities on that context adds up to exactly one, that is, $\sum_{i \in \text { clique/context }} P\left(c, v_{i}\right)=1$. Thereby, probabilities and expectations of simultaneously co-measurable observables, represented by graph vertices within cliques or contexts, obey Specker's exclusivity principle and "behave classically." It might be challenging to motivate "quantum type" probabilities and their convex expansion, the theta body (Grötschel et al. 1986), by the very assumptions such as exclusivity (Cabello et al. 2014; Cabello 2019).

A very similar measure on the closed subspaces of Hilbert space, satisfying Specker's exclusivity principle and additivity, had been proposed by Gleason Gleason (1957), first and second paragraphs, p. 885: "A measure on the closed subspaces means a function $\mu$ which assigns to every closed subspace a nonnegative real number such that if $\left\{A_{i}\right\}$ is a countable collection of mutually orthogonal subspaces having closed linear span $B$, then $\mu(B)=\sum_{i} \mu\left(A_{i}\right)$. It is easy to see that such a measure can be obtained by selecting a vec- 
tor $v$ and, for each closed subspace A, taking $\mu(A)$ as the square of the norm of the projection of $v$ on A." Gleason's derivation of the quantum mechanical Born rule (Born 1926, Footnote 1, Anmerkung bei der Korrektur, p. 865) operates in dimensions higher than two and allows also mixed states, that is, outcomes of nonideal measurements. However, mixed states can always be "completed" or "purified" (Nielsen and Chuang 2010, Section 2.5, pp. 109-111) (and thus outcomes of nonideal measurements made ideal Cabello 2019) by the inclusion of auxiliary dimensions.

\section{Quasiclassical analogues of entanglement}

In what follows, classical analogs to entangled states will be discussed. These examples are local. They are based on Schrödinger's observation that entanglement among pairs of particles is associated with, or at least accompanied by, joint or relational (Zeilinger 1999) properties of the constituents, whereas nonentangled states feature individual separate properties of the pair constituents (Schrödinger 1935a, b, 1936). (For early similar discussions in the measurement context, see von Neumann 1955, Section VI.2, p 426, pp 436-437 and London and Bauer 1939; London and Edmond 1983.)

\subsection{Partitioning of state space}

Wrights generalized urn model (Ron 1978; Wright 1990), in a nutshell, is the observation of black balls, on which multiple colored symbols are painted, with monochromatic filters in only one of those colors. Complementarity manifests itself in the necessity of choice of the particular color one observes: one may thereby obtain knowledge of the information encoded in this color, but thereby invariable loses messages encoded in different colors. A typical example is the logic $L_{12}$ encoded by the partition logic enumerated in (1) and depicted in Fig. 1(a): suppose that there are 4 ball types and two colors on black backgrounds:

- ball type 1 is colored with orange a and blue a;

- ball type 2 is colored with orange $b$ and blue $c$;

- ball type 3 is colored with orange $\mathrm{c}$ and blue $\mathrm{b}$;

- ball type 4 is colored with orange $\mathrm{c}$ and blue $\mathrm{c}$.

Suppose an urn is loaded with balls of all four types. Suppose further that an agent's task is to draw one ball from the urn and, by observing this ball, to find which type it is. Of course, if the observer is allowed to look at both colors simultaneously, this would allow to single out exactly one ball type. But that maximal resolution can no longer be maintained in experiments restricted to one of the two colors. Any one of such two experiment varieties could resolve different, com-
Table 1 Six subensembles $E_{1}-E_{6}$ of the set $\{00,01,10,11\}$ with the following properties: $E_{1}=\{00,01\}$ encodes the first digit being 0; $E_{2}=\{10,11\}$ encodes the first digit being $1 ; E_{3}=\{00,10\}$ encodes the second digit being $0 ; E_{4}=\{01,11\}$ encodes the second digit being $1 ; E_{5}=\{00,11\}$ encodes the first and the second digit being equal; $E_{6}=\{01,10\}$ encodes the first and the second digit being different

\begin{tabular}{ccccc}
\hline \hline sample & ball type 1 & ball type 2 & ball type 3 & ball type 4 \\
\hline$E_{1}$ & 00 & 01 & & \\
$E_{2}$ & & & 10 & 11 \\
$E_{3}$ & 00 & & 10 & 11 \\
$E_{4}$ & & 01 & & 11 \\
\hline$E_{5}$ & 00 & & & \\
$E_{6}$ & & 01 & 10 & \\
\hline \hline
\end{tabular}

Table 2 Subensembles $\left(E_{5}\right)^{2}$ and $\left(E_{6}\right)^{2}$ of the set $\{0000,0001$, 0010, 0011, 0100, 0101, 0110, 0111, 1000, 1001, 1010, 1011, $1100,1101,1110,1111\}$ with the following properties: $E_{5}=$ $\{0000,0011,1100,1111\}$ encodes the first and the second pair, as well as the third and the fourth pair of digits being equal; $E_{6}=$ $\{0101,0110,1001,1010\}$ encodes the first and the second pair, as well as the third and the fourth pair of digits being different

\begin{tabular}{lcccc}
\hline \hline sample & ball type 1 & ball type 2 & ball type 3 & ball type 4 \\
\hline$\left(E_{5}\right)^{2}$ & 0000 & 0011 & 1100 & 1111 \\
$\left(E_{6}\right)^{2}$ & 0101 & 0110 & 1001 & 1010 \\
\hline \hline
\end{tabular}

plementary properties: looking at the drawn ball with orange glasses, the agent is able to resolve between balls (i) of type 1 associated with the symbol a; (ii) of type 2 associated with the symbol b; and (iii) of type 3 or 4 associated with the symbol c. The resolution between type 3 and 4 balls is lost. Alternatively, by looking at the drawn ball with blue glasses the agent is able to resolve between balls (i) of type 1 associated with the symbol a; (ii) of type 3 associated with the symbol b; and (iii) of type 2 or 4 associated with the symbol $\mathrm{c}$, that is, for the color blue the resolution between type 2 and 4 balls is lost. In any case, the state of ball type is partitioned in different ways, depending on the color of the filter. (Similar considerations apply for initial state identification problems on finite automata.)

\subsection{Relational encoding}

Tables 1 and 2 enumerate a relational encoding among two or more colors not dissimilar to Peres' detonating bomb model (Peres 1978). Suppose that an urn is loaded with balls of the type occurring in subensemble $E_{6}$ of Table 1 . The observation of some symbol $s \in\{0,1\}$ in green implies the (counterfactual) observation of the same symbol $s$ in red, and vice versa. Table 2 is just an extension to two colors per observer, and an urn loaded with subensembles $\left(E_{6}\right)^{2}$. Agent Alice draws a ball from the urn and looks at it with 
her red (exclusive) or blue filters. Then, Alice hands the ball over to Bob. Agent Bob looks at the ball with his green (exclusive) or orange filters. This latter scenario is similar to a Clauser-Horne-Shimony-Holt scenario of the EinsteinPodolsky-Rosen type, except that the former is totally local and its probabilities derived from the convex hull of the dispersion-free weights can never violate classical bounds, whereas the latter one may be (and hopefully is) nonlocal, and its performance with a quantum resource violates the classical bounds.

\section{Partition logic freak show}

Let us, for a moment, consider partition logics not restraint by low-dimensional faithful orthogonal representability, but with a separable set of two-valued states (with the exception of the logic depicted in Fig. 2f). These have no quantum realization. Yet, due to the automaton logic, they are intrinsically realized in Turing universal environments.

An example of such a structure is Wright's triangle logic (Wright 1990, Figure 2, p. 900) depicted in Fig. 2a (see also Dvurečenskij et al. 1995, Fig. 6, Example 8.2, pp. 414,420,421). As mentioned earlier, together with 4 dispersion-free weights it allows another weight $\frac{1}{2}$ on its intertwining vertices. Figure $2 b$, c depicts a square logic, the latter one being formed by a pasting of two triangle logics along a common leg. Figure $2 d$, e depicts pentagon logic with one and three inner cliques/contexts; the latter one realizing a true-implies-four-times-true configuration for $\{3\}$. Figure $2 \mathrm{f}$ has no partition logic representation, as its 5 dispersion-free weights cannot separate three atoms realized by $\{1\}$ and $\{2,3\}$, as well as two atoms realized by $\{2,4\}$ and $\{4,5\}$, respectively.

\section{Identical graphs realizable by different physical resources require different probability types}

It is important to emphasize that both scenarios-the classical generalized urn scenario as well as the quantized onefrom a graph theoretical point of view, operate with identical (exclusivity) graphs (e.g., Figure 1 in both References Fritz et al. 2013; Cabello et al. 2014). The difference is the representation of these graphs: the quantum case has a faithful orthogonal representation in some finite-dimensional Hilbert space, whereas the classical case in terms of a generalized urn model has a set-theoretic representation in terms of partitions of some finite set.

Generalized urn models and automaton logics are models of partition logics which are capable of complementarity yet fail to render (quantum) value indefiniteness. They are impor-

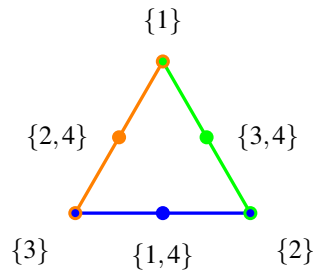

(a)

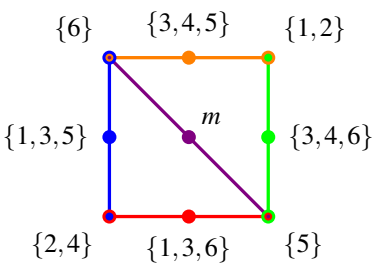

(c)

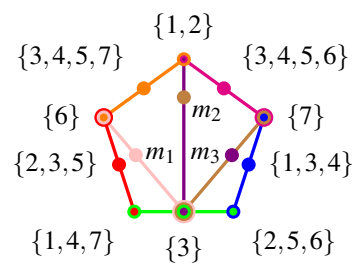

(e)

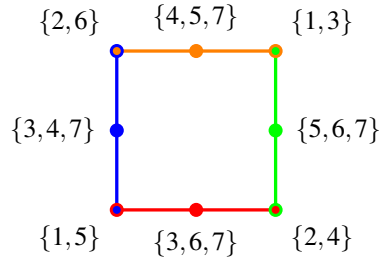

(b)

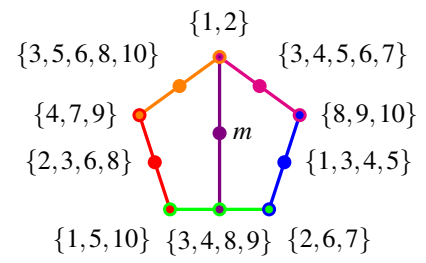

(d)

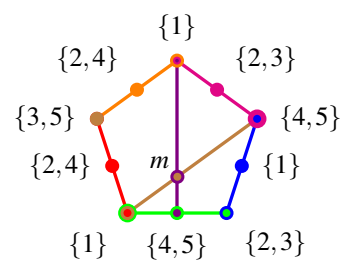

(f)
Fig. 2 Greechie orthogonality diagram of partition logic realizations of a Wright's triangle logic with a partition logic realization (Wright 1990; Dvurečenskij et al. 1995); b a square logic from the cyclic pasting of four three-atomic edges/cliques/contexts $K_{3}$; c a combo of triangle logics pasted along a common edge with a partition logic realization (Dvurečenskij et al. 1995, Example 8.3, pp. 421,422), with $m=\{1,2,3,4\}$; d pentagon logic with inner edge, with a partition logic realization, and $m=\{5,6,7,10\}$; e pentagon logic with three inner edges, with a partition logic realization, and $m_{1}=\{1,2,4,5,7\}$, $m_{2}=\{4,5,6,7\}, m_{3}=\{1,2,4,5,6\} ; \mathbf{f}$ pentagon logic with two inner edges and $m=\{2,3\}$, without a partition logic realization since the vertex representation generated from the 5 dispersion-free weights is highly degenerate and nonseparating

tant for an understanding of the "twilight zone" spanned by nonclassicality (nondistributivity, nonboolean logics) and yet full value definiteness-one may call this a "purgatory"floating in-between classical Boolean and quantum realms.

It should be stressed that the algebraic structure of empirical logics, or graphs, does in general not determine the types of probability measures on them. For instance, a generalized urn loaded with balls rendering the pentagon structure, as envisioned by Wright, has probabilities different from the scheme of Grötschel, Lovász and Schrijver, which is based on orthogonal representations of the pentagon. Likewise, a geometric resource such as a "vector contained in a box" and "measured along projections onto an orthonormal basis" will not conform to probabilities induced by the convex hull of the dispersion-free weights - even if these weights are separating. Therefore, the particular physical resource-what 
is actually inside the black box-determines which type of probability theory is applicable.

Furthermore, partition logics which are not just a single Boolean algebra represent empirical configurations featuring complementarity. And yet they all have separating (Kochen and Specker 1967, Theorem 0) sets of two-valued states and thus are not "contextual" in the Specker sense (Specker 1960).

Acknowledgements Open access funding provided by TU Wien (TUW). This work was greatly inspired by Adán Cabello's insistence on graph theoretical importance for quantum mechanics and for his challenge to come up with a classical Einstein-Podolsky-Rosen type scenario.

\section{Compliance with ethical standards}

Conflicts of interest The author declares that he has no conflict of interest.

Open Access This article is distributed under the terms of the Creative Commons Attribution 4.0 International License (http://creativecomm ons.org/licenses/by/4.0/), which permits unrestricted use, distribution, and reproduction in any medium, provided you give appropriate credit to the original author(s) and the source, provide a link to the Creative Commons license, and indicate if changes were made.

\section{References}

Badzia̧g P, Bengtsson I, Cabello A, Granström H, Larsson J-A (2011) Pentagrams and paradoxes. Found Phys 41:414-423

Born M (1926) Zur Quantenmechanik der Stoßvorgänge. Zeitschrift für Physik 37:863-867

Bub J, Stairs A (2009) Contextuality and nonlocality in 'no signaling' theories. Found Phys 39:690-711

Bub J, Stairs A (2010) Contextuality in quantum mechanics: testing the Klyachko inequality. arXiv: 1006.0500

Cabello Adán (2012) Specker's fundamental principle of quantum mechanics. arXiv: 1212.1756

Cabello Adán (2019) Physical origin of quantum nonlocality and contextuality. arXiv: 1801.06347

Cabello A (2013) Simple explanation of the quantum violation of a fundamental inequality. Phys Rev Lett 110:060402 arXiv:1210.2988

Cabello A, Danielsen LE, López-Tarrida AJ, Portillo JR (2013) Basic exclusivity graphs in quantum correlations. Phys Rev A 88:032104 arXiv: 1211.5825

Cabello A, Severini S, Winter A (2014) Graph-theoretic approach to quantum correlations. Phys Rev Lett 112:040401 arXiv:1401.7081

Cabello A, Severini S, Winter A (2010) (non-)contextuality of physical theories as an axiom. arXiv: 1010.2163

Dvurečenskij A, Pulmannová S, Svozil K (1995) Partition logics, orthoalgebras and automata. Helv Phys Acta 68:407-428 arXiv: 1806.04271

Fritz T, Sainz AB, Augusiak R, Brask J Bohr, Chaves R, Leverrier A, Acín A (2013) Local orthogonality as a multipartite principle for quantum correlations. Nat Commun. arXiv: 1210.3018

Gleason AM (1957) Measures on the closed subspaces of a Hilbert space. J Math Mech 6:885-893

Greechie RJ (1971) Orthomodular lattices admitting no states. J Comb Theory Ser A 10:119-132
Grötschel M, Lovász L, Schrijver A (1986) Relaxations of vertex packing. J Comb Theory Ser B 40:330-343

Haemers W (1979) On some problems of Lovász concerning the Shannon capacity of a graph. IEEE Trans Inf Theory 25:231-232

Halmos PR (1958) Finite-dimensional vector spaces, undergraduate texts in mathematics. Springer, New York

Henson J (2012) Quantum contextuality from a simple principle? arXiv: 1210.5978

Klyachko AA, Can MA, Binicioğlu S, Shumovsky AS (2008) Simple test for hidden variables in spin-1 systems. Phys Rev Lett 101:020403 arXiv:0706.0126

Kochen S, Specker EP (1967) The problem of hidden variables in quantum mechanics. J Math Mech 17:59-87

Liang Y-C, Spekkens RW, Wiseman HM (2011) Specker's parable of the overprotective seer: a road to contextuality, nonlocality and complementarity. Phys Rep 506:1-39 (2011). arXiv:1010.1273

Liang Y-C, Spekkens RW, Wiseman HM (2017) Erratumto "Specker's parable of the over-protective seer: a road to contextuality, nonlocality and complementarity" [phys. rep. 506 (2011) 1-39]. Phys Rep 666: 110-111 (2017). arXiv:1010.1273

London F, Bauer E (1939) La theorie de l'observation en mécanique quantique; No. 775 of Actualités scientifiques et industrielles: Exposés de physique générale, publiés sous la direction de Paul Langevin (Hermann, Paris, 1939) english translation in (London and Bauer 1983)

London F, Bauer E(1983) The theory of observation in quantum mechanics. In: Quantum theory and measurement, Princeton University Press, Princeton, NJ, 1983, pp 217-259, consolidated translation of French original (London and Bauer 1939)

López Tarrida AJ (2014) Quantum correlations and graphs. Ph.D. thesis, Universidad de Sevilla

Lovász L (1979) On the Shannon capacity of a graph. IEEE Trans Inf Theory 25:1-7

Lovász L, Saks M, Schrijver A (1989) Orthogonal representations and connectivity of graphs. Linear Algebra Appl 114:439-454

Moore EF (1956) Gedanken-experiments on sequential machines (AM-34). In: Shannon CE, McCarthy J (eds) Automata studies. Princeton University Press, Princeton, NJ, pp 129-153

Nielsen MA, Chuang IL (2010) Quantum computation and quantum information. Cambridge University Press, Cambridge

Parsons TD, Pisanski T (1989) Vector representations of graphs. Discret Math 78:143-154

Peres A (1978) Unperformed experiments have no results. Am J Phys 46:745-747

Ron W (1978) The state of the pentagon. A nonclassical example. In: Marlow AR (ed) Mathematical foundations of quantum theory. Academic Press, New York, pp 255-274

Schaller M, Svozil K (1994) Partition logics of automata. Il Nuovo Cimento B 109:167-176

Schaller M, Svozil K (1995) Automaton partition logic versus quantum logic. Int J Theor Phys 34:1741-1750

Schaller M, Svozil K (1996) Automaton logic. Int J Theor Phys 35:911940

Schrödinger E (1935) Die gegenwärtige Situation in der Quantenmechanik. Naturwissenschaften 23:807-812, 823-828, 844-849

Schrödinger E (1935) Discussion of probability relations between separated systems. Math Proc Camb Philos Soc 31:555-563

Schrödinger E (1936) Probability relations between separated systems. Math Proc Camb Philos Soc 32:446-452

Sloane NJA (2018) A000110 Bell or exponential numbers: number of ways to partition a set of $n$ labeled elements. Formerly m1484 $\mathrm{n} 0585$. The on-line encyclopedia of integer sequences

Solís-Encina A, Portillo JR (2015) Orthogonal representation of graphs. arXiv: 1504.03662

Specker E (2009) Ernst Specker and the fundamental theorem of quantum mechanics, video by Adán Cabello, recorded on June 17, 2009 
Specker E (1960) Die Logik nicht gleichzeitig entscheidbarer Aussagen. Dialectica 14:239-246 arXiv:1103.4537

Svozil K (2001) On generalized probabilities: correlation polytopes for automaton logic and generalized urn models, extensions of quantum mechanics and parameter cheats. arXiv:quant-ph/0012066

Svozil K (2016) Generalized event structures and probabilities. In: Burgin M, Calude CS (eds) Information and complexity, world scientific series in information studies: vol. 6, World Scientific, Singapore, 2016, Chap. Chapter 11, pp 276-300. arXiv:1509.03480

Svozil Karl (2018) Kolmogorov-type conditional probabilities among distinct contexts. arXiv: 1903.10424

Svozil K (1993) Randomness \& undecidability in physics. World Scientific, Singapore

Svozil K (2005) Logical equivalence between generalized urn models and finite automata. Int $\mathrm{J}$ Theor Phys 44:745-754 arXiv:quant-ph/0209136

Svozil K (2006) Staging quantum cryptography with chocolate balls. Am J Phys 74:800-803 arXiv:physics/0510050

Svozil K (2009) Contexts in quantum, classical and partition logic. In: Engesser K, Gabbay DM, Lehmann D (eds) Handbook of quantum logic and quantum structures. Elsevier, Amsterdam, pp 551-586 arXiv:quant-ph/0609209
Svozil K (2014) Non-contextual chocolate ball versus value indefinite quantum cryptography. Theoret Comput Sci 560:82-90 arXiv:0903.0231

Svozil K (2018) Physical (a)causality, fundamental theories of physics, vol 192. Springer, Cham

von Neumann J (1955) Mathematical foundations of quantum mechanics. Princeton University Press, Princeton, NJ, German original in (von Neumann 1996)

von Neumann J (1931) Über Funktionen von Funktionaloperatoren. Ann Math (Ann Math) 32:191-226

von Neumann J (1996) Mathematische Grundlagen der Quantenmechanik, 2nd edn. Springer, Berlin

Wright R (1990) Generalized urn models. Found Phys 20:881-903

Zeilinger A (1999) A foundational principle for quantum mechanics. Found Phys 29:631-643

Publisher's Note Springer Nature remains neutral with regard to jurisdictional claims in published maps and institutional affiliations. 